\title{
MAPAS DE PROGRESO DEL APRENDIZAJE (MPA) Y RUTAS DE APRENDIZAJE (RA) EN PERÚ - 2013
}

LEARNING PROGRESS MAP (MPA) AND ROUTE OF LEARNING (RL) IN PERU - 2013

\author{
Marino Latorre $A .^{1}$ \\ Graduate School Director from Marcelino Champagnat University \\ LIMA - PERU
}

(RECIBIDO 12/03/2013 - ACEPTADO 06/06/2013)

\section{RESUMEN}

El presente artículo trata de los Mapas de Progreso de Aprendizaje (MPA) y de las Rutas de Aprendizaje (RA) implementados ambos por el Ministerio de Educación del Perú en el 2013. Ambos instrumentos están diseñados para fortalecer los aprendizajes de los estudiantes, proporcionar indicadores sobre la calidad de los aprendizajes de los estudiantes al final de los ciclos señalados y dar orientaciones a los docentes.

Se presentan las bases teóricas de los MPA y se hace un análisis crítico de ambos instrumentos, su origen, su validez y las posibles lecturas que se pueden hacer de los mismos, en función del paradigma que utilice el docente. Al final se expone de forma sintética el Paradigma socio-cognitivo-humanista como modelo pedagógico adecuado para la utilización correcta de los instrumentos estudiados.

Palabras clave: mapas de progreso del aprendizaje, rutas de aprendizaje, paradigma sociocognitivo-humanista

\begin{abstract}
This article discusses the Learning Progress Maps (LPM) and Learning Paths, (LP) implemented both by the Ministry of Education of Peru in 2013. Both instruments are designed to strengthen student learning process, to provide indicators to students learning quality therefore at the end of the cycles identified and provide guidance to teachers.

We present the theoretical basis of the LPM and a critical analysis of both instruments are done, its origin, its validity and possible readings that can be made of it, depending on the paradigm used by the teacher. At the end it is synthetically exposed the Socio-Cognitive humanist Paradigm as a pedagogical model suitable for the proper use of the instruments studied.
\end{abstract}

Keywords: learning progress maps, learning paths, socio-cognitive-humanist paradigm 
Mapas de progreso del aprendizaje (MPA) y rutas de aprendizaje (RA) en Perú - 2013

\section{INTRODUCCIÓN}

Con el fin de reducir la brecha entre el Curriculum prescrito y el Curriculum desarrollado en las escuelas, el MINEDU del Perú (2013) ha creído necesario identificar y definir los aprendizajes fundamentales que los estudiantes de EBR deben lograr. Para ello, además del Diseño Curricular Nacional (2008) (DCN) ha implementado los Mapas de Progreso de Aprendizaje (MPA), que expresan el nivel de progreso que deben alcanzar los estudiantes al final de cada ciclo y las Rutas de aprendizaje (RA) que son herramientas de apoyo a los docentes para el logro de los aprendizajes de los estudiantes.

El DCN responde a esta pregunta: ¿Qué deben aprender los estudiantes durante los estudios de EBR?

Los MPA responden a esta: ¿Qué indicadores hay que observar para saber si se han producido los aprendizajes? Permiten determinar si se han logrado los aprendizajes fundamentales a lo largo de los años de escolaridad.

Las RA definen las capacidades-destrezas que hay que desarrollar en cada área e indican cómo se debe enseñar. Ofrecen al profesor orientaciones didácticas y metodológicas para que el estudiante pueda aprender. Responde al ¿cómo actuar en el aula para desarrollar las capacidades-destrezas y aprender contenidos? Incluyen además materiales educativos que sirven de apoyo en el proceso de aprendizaje del estudiante y los docentes.

Las competencias básicas son las mismas para toda la EBR. Varían los indicadores -destrezas- que dan cuenta de los logros en cada una de las áreas, en función de la complejidad de los contenidos a que se aplican las destrezas o indicadores.

Hasta el primer semestre del 2013 el MINEDU había publicado las rutas de aprendizaje de tres áreas: Comunicación, Matemáticas y Educación para la Ciudadanía. Al término del 2013 se aprobará el Marco Curricular que definirá los aprendizajes fundamentales. Se irán alineando los diferentes instrumentos que componen el sistema curricular: marco curricular, mapas de progreso, rutas de aprendizaje y los currículos regionales.

\section{CONCEPTO DE MAPAS DE PROGRESO DE APRENDIZAJE (MPA)}

Los MPA trabajan con estándares que son "descripciones claras y específicas de las habilidades y conocimientos que el estudiante debe adquirir en un momento determinado de su escolaridad" (Kendal, 2001). Muestran el progreso del estudiante en su recorrido de aprendizaje. Es una manera de estandarizar el aprendizaje y progreso de los estudiantes. Según Forster (2007), los MPA responden a esta pregunta, “¿qué esperamos que los estudiantes sepan, comprendan y sean capaces de hacer" en un momento determinado de su escolaridad?

Los mapas de progreso son la descripción general de la secuencia de conocimientos que un niño debiera alcanzar en un determinado ciclo del nivel de aprendizaje en 
que se encuentre. Permiten al profesor focalizar su mirada en los aprendizajes centrales, de modo que pueda observar cuán lejos o cerca están sus estudiantes del nivel de logro, para poder orientar su pedagogía. Los niveles de logro vendrían a ser una descripción más fina del mismo aprendizaje.

De esta manera, se conecta el proceso de los niños con una historia escolar, donde los profesores compartan con sus colegas la información de cada estudiante. En los MPA se mira al estudiante a lo largo de su formación y no solo año a año; la mirada tiene un énfasis en la progresión y en mejorar la calidad de la educación. (Gysling, J., 2008, del Ministerio de Educación de Chile).

Según IPEBA (2013) un mapa de progreso describe la dirección del crecimiento del aprendizaje a lo largo de la trayectoria escolar, en cada una de las competencias clave de las distintas áreas curriculares.

- Esta descripción muestra en forma concisa la secuencia típica del aprendizaje en niveles.

- La secuencia típica está basada en evidencias.

Los MPA los diseña el Ministerio de Educación a partir del DCN. Son una ayuda para los profesores, a fin de que puedan identificar y definir los aprendizajes fundamentales que todos los estudiantes tienen derecho a lograr, desarrollar los programas del DCN y proponer actividades que favorezcan la comprensión y desarrollo de las habilidades que el DCN propone para cada nivel y ciclo de estudios. Son una secuencia de aprendizaje y un marco que permite monitorear el progreso de los estudiantes. Para ello los MPA:

- Deben ser claros y orientadores de la práctica docente

- Deben concretar las intenciones educativas en términos de competencias

- Deben permitir "alinear" el currículum y los estándares y presentarlos en progresión.

Los MPA se concretan y especifican a través de las Rutas de aprendizaje, entendidas como actividades concretas y adaptadas a los ciclos y áreas para garantizar que los aprendizajes se han conseguido al finalizar los ciclos correspondientes. Son como modelos de actividades que debe proponer el profesor para garantizar que los estudiantes desarrollan las capacidades-competencias y aprenden los contenidos de las distintas áreas.

Según el MINEDU cada uno de los instrumentos curriculares debe abordar el aprendizaje a partir de diferentes funciones:

- El DCN establece ¿qué deben aprender los estudiantes? Definen los aprendizajes fundamentales, es decir, los aprendizajes que se espera logren todas y todos los estudiantes del país y las capacidades y valores que deben desarrollar.

- Los MPA indican ¿qué se debe observar y con qué criterios debe observar el docente? Ofrecen indicadores precisos para observar el logro de los aprendizajes 
fundamentales a lo largo de la escolaridad. Describen qué es lo central que el docente debe observar en el aprendizaje de los estudiantes para poder monitorear y apoyar el progreso de aprendizaje.

- Las RA explican ¿cómo se debe y puede enseñar? Ofrecen orientaciones pedagógicas y didácticas para el logro de los aprendizajes fundamentales. (MINEDU (2013). Las rutas se han construido a partir de los mapas de progreso que expresan los estándares de desempeño que debe lograr cada estudiante al término de cada ciclo de la educación básica regular. Las rutas de aprendizaje no dan recetas que hay que seguir de manera ciega y rígida, por el contrario ofrecen orientaciones que cada docente podrá adecuar a su realidad, haciendo uso de sus saberes pedagógicos y su creatividad.

- Libros de texto y materiales pedagógicos ¿cómo apoyar el desarrollo de los aprendizajes del currículum en el aula? Desarrollan los aprendizajes definidos en el marco curricular para apoyar el trabajo de estudiantes y docentes.

Los MPA y las RA en ningún caso pueden considerarse como sustitutivos del DCN, ni constituyen un nuevo diseño curricular. Son una referencia ideal de los cursos asociados a los niveles de progreso del estudiante. Posibilitan el trabajo coordinado de los profesores de la misma área en los distintos grados y ciclos. Por otra parte sirven como criterios e indicadores de evaluación. Los MPA indican el camino o ruta de aprendizaje de las distintas áreas, permiten identificar la situación cognitiva de cada estudiante y sus distintos ritmos de aprendizaje en un curso y grado determinado y las Rutas de aprendizaje cómo recorrer el camino para obtener el máximo aprovechamiento.

Según Bransford, J. D. et al. (2000) los docentes identifican los conocimientos que el estudiante ya posee y a partir de ahí trabajan.

- La enseñanza se debe orientar al aprendizaje de conocimientos organizados y a una comprensión profunda de los conceptos y su aplicabilidad.

- Es fundamental en el proceso desarrollar en el estudiante la capacidad para monitorear y regular el proceso de su propio aprendizaje - autorregulación -.

Los MPA que han sido propuestos por el MINEDU siguen la propuesta del Ministerio de Educación de Chile. En Chile hay 12 años de EBR - desde $1^{\circ}$ de Primaria a $6^{\circ}$ de Secundaria --, sin contabilizar la educación preescolar de 0-5 años. Por esta razón divide la Educación Básica en 7 niveles que corresponden a la división de los años de aprendizaje. Es una división razonable y lógica pues se ha hecho en función de los años y niveles de escolaridad.

Siguiendo este modelo el IPEBA del Perú -en conexión con el MINEDU establece también siete niveles de aprendizaje, desde Educación Pre-Inicial hasta $5^{\circ}$ de Secundaria. Cada uno de los niveles coincide, aproximadamente, con dos años de escolaridad; los siete niveles corresponden a los grados que indicamos a continuación. Pero como en Perú solo hay 11 años de escolaridad entre $1^{\circ}$ de Primaria y $5^{\circ}$ de Secundaria podemos observar que los niveles propuestos no coinciden con 


\begin{tabular}{|c|c|c|}
\hline N. $^{\circ}$ de nivel de MPA & Ciclos del DCN & Grados \\
\hline Nivel 7 & --- & Por encima de Nivel 6 \\
\hline Nivel 6 & VII & $3^{\text {o }}, 4^{\circ}$ y $5^{\circ}$ Secundaria \\
\hline Nivel 5 & VI & $1^{\text {o }}$ y $2^{\circ}$ Secundaria \\
\hline Nivel 4 & V & $5^{\circ}$ y $6^{\circ}$ Primaria \\
\hline Nivel 3 & IV & $3^{\circ}$ y $4^{\circ}$ Primaria \\
\hline Nivel 2 & III & $1^{\circ}$ y $2^{\circ}$ Primaria \\
\hline Nivel 1 & II & $\begin{array}{c}\text { Previo al Nivel 2 Pre-Inicial } \\
(0-3 \text { años }) \text { e Inicial (3-5 años) }\end{array}$ \\
\hline
\end{tabular}

Fuente: Instituto Peruano de Evaluación Acreditación y Certificación de la Calidad de la educación Básica (IPEBA). (2013). Programa de estándares de aprendizaje. Lima, Perú.

los del DCN. Es razonable, pues los niveles indican los aprendizajes que corresponden al último grado de los que se encuentran en el nivel. En el caso del nivel séptimo no existe el $6^{\circ}$ curso de Secundaria para poderlo colocar en ese nivel. El esquema que propone IPEBA queda así:

Como se ha señalado, los MPA describen la secuencia en que comúnmente progresa el estudiante en su aprendizaje en determinadas áreas o competencias clave que se consideran fundamentales en la formación. Esta descripción está hecha de un modo conciso y claro, para que todos (docentes, estudiantes, directores y familia) puedan compartir esta visión sobre cómo progresa el aprendizaje a través de los años de escolaridad. Se pretende que los MPA se constituyan en un marco de referencia para dialogar sobre el aprendizaje que se espera y que, a partir de trabajos prácticos realizados por los mismos estudiantes, -- Rutas de aprendizaje -- sirvan para juzgar dónde se encuentran en esta progresión, para determinar hacia dónde deben avanzar.

Analizando los ejemplos de los mapas de progreso que presentamos, nos parece que el Nivel 1, que corresponde al Ciclo II de Educación Inicial del Perú, contiene habilidades excesivas para niños de esa edad, pues indican que el estudiante debe saber leer y comprender lo que lee a la edad de 5 años. Tengo dudas de que sea una realidad para Chile u otros países, pero creo que no lo puede ser para la inmensa mayoría de los niños de esa edad del Perú. La razón es que, a esa corta edad, los niños están comenzando a conocer los signos gráficos, a decodificarlos mecánicamente, etc.; dudo que puedan hacer inferencias. 
Como se ve todo mapa de progreso contiene tres aspectos:

a) Dominio: área de aprendizaje a que hace referencia

b) Niveles: grados

c) Dimensiones - descripción

El siguiente es un ejemplo de los enunciados de los siete niveles de un Mapa de Progreso, que en este caso corresponde al Mapa de Progreso de lectura del sector de Lenguaje y Comunicación. Como puede observarse, en este Mapa se describe de una manera sintética lo medular del desarrollo de la comprensión lectora de Primero de Educación Primaria al último año de Secundaria, del Mineduc de Chile (2008).

\begin{tabular}{|l|l|}
\hline \multicolumn{2}{|c|}{ Ejemplo de un mapa de progreso de Comprensión lectora } \\
\hline Nivel 7 & $\begin{array}{l}\text { Lee comprensivamente variados tipos de texto de carácter analítico } \\
\text { y reflexivo. (Analizar y relacionar con otros conocimientos) } \\
\text { sobresaliente } \\
\text { Interpreta y re-interpreta, a partir de énfasis y matices, sentidos } \\
\text { globales del texto o de partes significativas del mismo, que expresan } \\
\text { ambigüedades, contradicciones o posturas poco claras. (Identificar } \\
\text { e interpretar, evaluar-valorar) } \\
\text { Evalúa la calidad del texto y la pertinencia de su estructura textual, } \\
\text { estilo y coherencia interna.(Evaluar- valorar, iopinar?) }\end{array}$ \\
\hline Nivel 6 & $\begin{array}{l}\text { Lee comprensivamente variados tipos de texto que le permiten } \\
\text { construir diferentes visiones del mundo. Interpreta y reinterpreta } \\
\text { sentidos globales del texto a partir de inferencias complejas e in- } \\
\text { formación del contexto sociocultural de su producción. Identifica } \\
\text { recursos expresivos que potencian el sentido general de la obra. } \\
\text { Evalúa la validez de los argumentos o planteamientos presentes } \\
\text { en los textos }\end{array}$ \\
\hline Nivel 5 & $\begin{array}{l}\text { Lee comprensivamente textos con variadas estructuras convencio- } \\
\text { nales y no convencionales, que abordan temas de diversos ámbitos. } \\
\text { Interpreta el sentido global del texto según las posibles perspectivas } \\
\text { del emisor o del receptor. Evalúa lo leído, comparándolo con su } \\
\text { postura o la de otros, frente al tema. }\end{array}$ \\
\hline
\end{tabular}




\begin{tabular}{|l|l|}
\hline Nivel 4 & $\begin{array}{l}\text { Lee comprensivamente textos con estructuras variadas, con ele- } \\
\text { mentos complejos, que abordan temas de diversos ámbitos. Extrae } \\
\text { información explícita de elementos complementarios que }\end{array}$ \\
$\begin{array}{l}\text { precisan o amplían la información central. Interpreta sentidos de } \\
\text { detalles y de partes del texto y los relaciona con su sentido global. } \\
\text { Opina sobre lo leído, comparando el contexto sociocultural pre- } \\
\text { sentado en el texto con el propio o con la actualidad }\end{array}$ \\
\hline Nivel 3 & $\begin{array}{l}\text { Lee comprensivamente textos de estructuras variadas, con algunos } \\
\text { elementos complejos, que abordan temas de diversos ámbitos. Ex- } \\
\text { trae información explícita relevante distinguiéndola de la accesoria. } \\
\text { Infiere relaciones de causa, efecto y secuencia, integrando detalles } \\
\text { relevantes del texto. } \\
\text { Comprende el sentido global del texto, integrando la información } \\
\text { extraída. Opina sobre variados aspectos del texto, apoyándose en } \\
\text { información explícita e implícita, e integrando sus conocimientos } \\
\text { específicos sobre el tema }\end{array}$ \\
\hline Nivel 2 & $\begin{array}{l}\text { Lee comprensivamente textos de estructura simple que abordan } \\
\text { contenidos reales o imaginarios, algunos de los cuales pueden ser } \\
\text { poco familiares. Extrae información explícita, distinguiéndola de } \\
\text { otras próximas y semejantes. Infiere relaciones de causa, efecto } \\
\text { y secuencia referidas a información central del texto. Compren- } \\
\text { de el sentido global del texto integrando información explícita e } \\
\text { implícita. Opina sobre contenidos de lo leído, apoyándose en la } \\
\text { información extraída }\end{array}$ \\
\hline $\begin{array}{l}\text { Lee comprensivamente textos breves y simples, que abordan conte- } \\
\text { nidos reales o imaginarios que le son familiares. Extrae información } \\
\text { explícita evidente. Realiza inferencias claramente sugeridas por el } \\
\text { texto. Comprende el sentido global a partir de información desta- } \\
\text { cada en el texto. Da sus opiniones sobre lo leído, apoyándose en } \\
\text { información explícita y en inferencias realizadas. }\end{array}$ \\
\hline
\end{tabular}

Fuente: Orientaciones para el uso de los Mapas de Progreso del Aprendizaje. Un material de apoyo para el trabajo docente. (2008). Gobierno de Chile. (p.23)

http://es.scribd.com/doc/84773499/Orientacion-Uso-Mapas-de-Progreso. 
Presentamos el mismo Mapa de Progreso realizado por el IPEBA - Perú - señalando los distintos aspectos indicados y las destrezas que subyacen en cada uno de los niveles.

Ejemplo de un mapa de progreso de Comprensión lectora

\begin{tabular}{|c|c|}
\hline Nivel 7 & $\begin{array}{l}\text { Lee comprensivamente textos con estructuras complejas, principal- } \\
\text { mente de naturaleza analítica y reflexiva, con vocabulario variado } \\
\text { y especializado. (Analizar y relacionar con otros conocimientos) } \\
\text { Interpreta y reinterpreta el texto a partir del análisis de énfasis } \\
\text { y matices intencionados reconociendo distintos temas y posturas } \\
\text { que aborda. Evalúa la efectividad y validez de los argumentos o } \\
\text { planteamientos del texto y del uso de los recursos textuales. } \\
\text { (Identificar e interpretar, evaluar-valorar). Explica la influencia de } \\
\text { los valores y posturas del autor en relación a la coyuntura socio- } \\
\text { cultural en la que el texto fue escrito. (Explicar y dar su opinión) }\end{array}$ \\
\hline Nivel 6 & $\begin{array}{l}\text { Lee comprensivamente textos con estructuras complejas que de- } \\
\text { sarrollan temas diversos con vocabulario variado y especializado. } \\
\text { (Analizar, explicar) } \\
\text { Integra información contrapuesta o ambigua que está en distintas } \\
\text { partes del texto. Interpreta el texto integrando la idea principal } \\
\text { con información relevante y de detalles. (Interpretar, comparar, } \\
\text { argumentar) } \\
\text { Evalúa la efectividad de los argumentos del texto y el uso de los } \\
\text { recursos textuales a partir de su conocimiento y del contexto so- } \\
\text { ciocultural en el que fue escrito. (Evaluar, relacionar) }\end{array}$ \\
\hline Nivel 5 & $\begin{array}{l}\text { Lee comprensivamente textos con estructuras complejas que de- } \\
\text { sarrollan temas diversos con vocabulario variado. } \\
\text { Integra información contrapuesta que está en distintas partes } \\
\text { del texto. Interpreta el texto integrando información relevante y } \\
\text { complementaria. Opina sobre aspectos variados, comparando el } \\
\text { contexto sociocultural presentado en el texto con el propio y explica } \\
\text { la intención de los recursos textuales integrando su conocimiento } \\
\text { y experiencia. }\end{array}$ \\
\hline
\end{tabular}




\begin{tabular}{|l|l|}
\hline Nivel 4 & $\begin{array}{l}\text { Lee comprensivamente textos con varios elementos complejos en } \\
\text { su estructura y que desarrollan temas diversos, con vocabulario } \\
\text { variado. (Interpretar y relacionar con otros conocimientos) } \\
\text { Extrae información e integra datos que están en distintas partes del } \\
\text { texto. Realiza inferencias locales a partir de información explícita } \\
\text { e implícita. Interpreta el texto integrando información relevante } \\
\text { y complementaria. (Identificar e inferir el tema central y otros) } \\
\text { Opina sobre aspectos variados del texto y explica la intención de } \\
\text { los recursos textuales a partir de su conocimiento y experiencia. } \\
\text { (Explicar y dar su opinión) }\end{array}$ \\
\hline Nivel 3 & $\begin{array}{l}\text { Lee comprensivamente textos que presentan estructura simple con } \\
\text { algunos elementos complejos y que desarrollan temas diversos con } \\
\text { vocabulario variado. } \\
\text { Extrae información poco evidente distinguiéndola de otras próxi- } \\
\text { mas y semejantes. Realiza inferencias locales a partir de infor- } \\
\text { mación explícita e implícita. Interpreta el texto seleccionando } \\
\text { información relevante. }\end{array}$ \\
\hline $\begin{array}{l}\text { Opina sobre sucesos e ideas importantes del texto y explica la } \\
\text { intención de los recursos textuales más comunes a partir de su } \\
\text { conocimiento y experiencia. }\end{array}$ \\
\hline $\begin{array}{l}\text { Lee comprensivamente textos de estructura simple que tratan temas } \\
\text { reales o imaginarios en los que predominan palabras conocidas e } \\
\text { ilustraciones que apoyan las ideas centrales. } \\
\text { Extrae información poco evidente distinguiéndola de otra semejan- } \\
\text { te y realiza inferencias locales a partir de información explícita. } \\
\text { Interpreta el texto relacionando información recurrente. } \\
\text { Opina sobre sucesos e ideas importantes del texto a partir de su } \\
\text { propia experiencia. }\end{array}$ \\
\hline $\begin{array}{l}\text { Lee textos de estructura simple que tratan temas reales o ima- } \\
\text { ginarios que le son cotidianos, en los que predominan palabras } \\
\text { conocidas y que se acompanan con ilustraciones. } \\
\text { Construye hipótesis y predicciones sobre la información contenida } \\
\text { en los textos y demuestra entendimiento de las ilustraciones y de } \\
\text { algunos símbolos escritos que transmiten información. } \\
\text { Expresa sus gustos y preferencias en relación a los textos leídos. } \\
\text { Utiliza algunas convenciones básicas de los textos escritos. }\end{array}$ \\
\hline
\end{tabular}

Fuente: IPEBA, (2013). Lima, Perú.

Como se puede apreciar es copia, casi textual, del de Chile. 


\section{BASES TEÓRICAS DE LOS MPA}

En la década de los años 80, Australia manejaba los mapas de progreso, también Inglaterra, Filipinas, en algunos estados de Canadá y EE. UU. Los MPA pretenden describir los distintos niveles de logro que pueden tener los estudiantes. En Chile la idea procede M. Forster (2007), asesora científica del Australian Council for Education Research. Según Forster (2007) las bases teóricas de los MPA son las siguientes:

a) Idea de crecimiento y mejora del aprendizaje. Los MPA expresan el progreso y desarrollo cognitivo del estudiante tanto para este como para el docente, pudiéndose tomar decisiones basadas en datos ciertos y de este modo impulsar el aprendizaje. El currículum debe estar diseñado como una red de conexiones más que como una secuencia de conocimientos aislados unos de otros.

Los MPA proporcionan un marco de referencia a los profesores. Si se leen e interpretan a la luz de un paradigma de corte socio-cognitivo pueden convertirse en un instrumento de renovación pedagógica del profesorado, que posibilitan un nuevo modo de aprender de forma significativa y funcional. Si se interpretan desde una perspectiva conductista, no.

b) Monitoreo del progreso de los estudiantes empleando un marco de referencia explícito. Los MPA son considerados como un conjunto ordenado y secuenciado de los resultados esperados de los estudiantes en un momento determinado de su proceso de aprendizaje, cumpliendo unos estándares. Tratan de hacer explícito el crecimiento cognitivo del estudiante y actúan como marcos de referencia para el docente y el estudiante. Los mapas de progreso se construyen con ejemplos reales de trabajos que tienen que hacer los estudiantes como forma de expresar su desempeño.

Este planteamiento es interesante, siempre que se considere el desarrollo cognitivo del estudiante como desarrollo de capacidades-destrezas -herramientas mentales, o pasos de pensamiento- en un modelo en que la enseñanza se subordine al aprendizaje. Así se puede hacer un seguimiento del qué aprende, cómo aprende y para qué aprende el estudiante. De este modo podemos hablar de un aprendizaje como un conjunto de procesos cognitivos y afectivos en fase de desarrollo que posibilitan el crecimiento y mejora de la inteligencia -teoría tridimensional de la inteligencia- (Díez, E. 2006). Estos procesos cognitivos y afectivos son observables y evaluables desde la evidencia.

c) Retroalimentación del proceso de aprendizaje-enseñanza haciendo posible la evaluación interna y externa. Los MPA son un sistema coherente para evaluar los logros de los estudiantes en todos los niveles; el nivel de logro del estudiante se compara con los mapas de progreso de su nivel que se convierten en criterios-indicadores de evaluación. La evaluación puede ser interna (recogida en el aula) y externa, realizada por organismos oficiales nacionales o internacionales. "Una vez que el docente comprende la naturaleza del crecimiento en un área de aprendizaje y ha establecido el nivel actual de logros del estudiante, 
podrá tomar decisiones, basadas en evidencias, acerca de cómo facilitar de mejor manera un mayor aprendizaje". (Forster, M. 2007)

El problema surge cuando se concretan tanto los contenidos en los MPA que el docente, arraigado en métodos tradicionales, puede llegar a la conclusión de que lo importante en el aprendizaje del estudiante son los contenidos, y no el desarrollo de las capacidades-destrezas como herramientas mentales, utilizando los contenidos como medios para desarrollarlas.

En este sentido afirmamos que las pruebas de evaluación - igual que las actividades - deben contener una habilidad (destreza) + contenido concreto + un método concreto.

\section{ANÁLISIS CRÍTICO DE LOS MAPAS DE PROGRESO DEL APRENDIZAJE (MPA)}

Los MPA han sido descritos como una herramienta auxiliar para establecer los estándares de desempeño, enfocados en la evaluación de aula con criterios preestablecidos y con expectativas definidas sobre lo que los estudiantes deben aprender o haber aprendido. (Forster, M., 2007, Mineduc de Chile).

Aunque los MPA sean un instrumento necesario para la evaluación formativa y la construcción de un necesario lenguaje común entre docentes al momento de describir el aprendizaje, analizándolos críticamente se observan problemas conceptuales en torno a la noción de aprendizaje (Salinas, I. 2012, 28). Los documentos que describen los MPA hablan del aprendizaje pero no contienen una noción explícita de lo que entienden por "el concepto de aprendizaje". Los MPA señalan una postura operacional que igual a los "niveles de desempeños" diferenciales con "niveles de aprendizaje", sin que estos se articulen en torno a una postura teórica explícita. Ello nos remite, de alguna manera, al "principio de verificación" de la teoría del positivismo lógico. Todo esto hace que quede muy poco espacio para una discusión que salga de los aspectos técnicos del uso de los MPA.

La principal crítica que se puede hacer a los MPA es que no hace explícito un fundamento epistemológico del aprendizaje (paradigma pedagógico) en que se sustentan. Por eso nos preguntamos: ¿Qué noción de aprendizaje hay detrás de los Mapas de Progreso del Aprendizaje? El MINEDU no lo explicita como tampoco lo hace en el DCN.

Cualquier estudioso del aprendizaje podrá observar que hay múltiples visiones sobre qué es el aprendizaje y cuál es la evidencia de que está ocurriendo o ha ocurrido. Así, el aprendizaje puede ser descrito desde el paradigma tradicional, personalista, conductista, hasta las bases del constructivismo cognitivo y socialcultural, como también desde las disciplinas neurocientíficas - neuroeducación --.

La definición de "aprendizaje" es una decisión que requiere una postura disciplinar que debe ser explícita, y ello se hace a un nivel teórico y otro operacional que permita la discusión y el consenso desde otras disciplinas. (Bransford, Brown \& Cocking, 2000). Esto no ocurre con los MPA, pues a pesar de su énfasis en el 
"aprendizaje", su foco no es el aprendizaje en sí mismo, sino "la observación de desempeños de los estudiantes en los diversos niveles" (Salinas, I. 2012, 29).

De esta forma, los MPA buscarían otorgar coherencia a la política multidimensional de estandarización educativa. Los MPA se articulan mejor con los estándares de aprendizaje que con el saber multidisciplinar en torno al aprendizaje. Se articulan aún menos con el saber pedagógico que guía a los docentes a responder a las distintas demandas de la enseñanza en distintos contextos. Este es un aspecto muy importante en un país, como el Perú, tan rico en multiculturalidad.

La base teórica del modelo MPA parte de la idea de crecimiento, entendido como progreso del estudiante en su aprendizaje, siendo los mapas de progreso descripciones del conocimiento adquirido o por adquirir y habilidad para adquirirlo. Según Román Pérez et al. (2008, 37 y ss.) esto nos remite a dos posibilidades:

\subsection{Lectura conductista de los mapas de progreso}

Los MPA muestran una coherente y detallada estrategia curricular con el fin de establecer una estandarización curricular en la realización de las tareas escolares. La creación, uso e interpretación de las tareas escolares está en la centralidad del poder estandarizador de los MPA.

Los MPA incluyen, de forma explícita, descripciones de desempeño en cada asignatura en forma de contenidos concretos por niveles, y así articulan los estándares académicos. Es decir, son una herramienta curricular que direcciona el juicio del docente respecto al cumplimiento de estándares.

Si los MPA se centran en el contendido (qué aprende el estudiante), quedando fuera el cómo aprende (métodos, técnicas, estrategias, habilidades, procesos mentales, etc.) y sobre todo el para qué aprende, estaríamos próximos a la taxonomía de Bloom que es de corte conductista. Los MPA mejoran la secuencia de los contenidos, pero existe el riesgo de que los profesores se queden ahí (en los contenidos solamente). Hace falta un cambio de marco de referencia (paradigma educativo) que haga posible el cambio de la práctica docente.

Es necesario diferenciar el "nivel del estudiante" del "nivel del desempeño del estudiante". Considerar el "nivel del estudiante" es difícil de medir si se les asocia una evidente carga emocional negativa y/o positiva en el clima del aula. Por otro lado, considerar el "nivel del desempeño del estudiante" implica centrar la atención en el proceso de realización de la tarea realizada por el estudiante y no solo en el logro conseguido.

Ejemplo de mapa de progreso de Ciencias Sociales en Chile, de Nivel $4^{\circ}$. Un estudiante ha logrado el Nivel $4^{\circ}$ en Ciencias Sociales cuando realiza las actividades siguientes:

- Sitúa acontecimientos de la historia, utilizando líneas de tiempo con décadas y siglos. 
- Establece relaciones entre la independencia del país y la de otros países latinoamericanos.

- Identifica fenómenos comunes en la historia de Latinoamérica.

- Propone ejemplos de rasgos culturales del Perú que comparte con otros países latinoamericanos, tales como idioma, costumbres y creencias.

- Identifica como fuentes históricas documentos que caracterizan aspectos culturales, económicos, políticos y/o sociales.

- Compara similitudes y diferencias entre los testimonios de distintos actores contemporáneos a los hechos relatados.

- Describe acontecimientos, personajes, sujetos y procesos históricos a partir de textos, imágenes, registros audiovisuales y objetos.

Como se puede observar este mapa de progreso trabaja con ciertas habilidades -destrezas- pero ignora en casi todos los caso el método que debe utilizar el estudiante y más aun los procesos mentales que debe seguir para desarrollar la destreza. Se observa que lo esencial ahí es el contenido, no el proceso de aprendizaje y la forma como desarrolla la habilidad o herramienta mental de aprendizaje.

Sigue la misma línea que el DCN $(2008,184)$, por ejemplo en $6^{\circ}$ de Primaria área de Comunicación que propone:

- Comprende textos sobre temas de actualidad.

- Relaciona el texto leído con su experiencia y otras realidades.

- Reconoce en los textos las normas de ortografía, las estructuras gramaticales, y el vocabulario...

- Lee textos que selecciona voluntariamente.

- Expresa su apreciación personal sobre los textos, presentando argumentos claros.

\subsection{Lectura socio-cognitiva de los MPA}

Desde este punto de vista lo esencial de los MPA son las destrezas-habilidades -entendidas como acciones mentales- existentes en los mapas de progreso que se convierten en indicadores de evaluación.

Las habilidades-destrezas que proponen los MPA en las rutas de aprendizaje son bastante sencillas, a veces confusas y no están secuenciadas por grado de dificultad y, a veces, las destrezas no corresponden con las competencias-capacidades que se han determinado para el área. Por otra parte se proponen como destrezashabilidades algunas que no lo son... Por ejemplo, para $2^{\circ}$ de Primaria en Comunicación-Comprensión lectora propone: decodificar (leer) identificar, relacionar, opinar, etc. de manera implícita está analizar. Lo que llama opinar, dudo que sea una destreza, más se parece "valorar". 
Mejor seleccionadas están las destrezas de Matemáticas; propone las siguientes: identificar, calcular, representar, cuantificar (contar), comparar, ordenar-secuenciar, procesar la información (resolver problemas), etc.

Román, M. et al. (2008, 35 y ss.) ha hecho el siguiente estudio crítico sobre los MPA:

3.2.1. Es un modelo pedagógico inductivo ya que parte del aprendizaje real de los estudiantes en el aula -lo que saben y lo que saben hacer- a partir de evidencias obtenidas por observación del aprendizaje. Este modelo pedagógico está relacionado con la Investigación-acción.

El inconveniente que tiene es que no específica el cómo hay que hacer (método), no habla de los procesos mentales que debe utilizar el estudiante para aprender a aprender y para atribuir significado a su aprendizaje y olvida el para qué aprender.

El aprendizaje característico de cada nivel se describe en palabras que expresan destrezas y se ilustra con desempeños y ejemplos de trabajo de los estudiantes.

Para reconocer el grado de desempeño proponemos algunos ejemplos tomados de los documentos del Ministerio de Educación de Chile (2008).

Ejemplo de Mapa de progreso de lectura - Nivel 4:

Lee comprensivamente textos con varios elementos complejos en su estructura y que desarrollan temas diversos, con vocabulario variado. Extrae información e integra datos que están en distintas partes del texto. Realiza inferencias locales a partir de información explícita e implícita. Interpreta el texto integrando información relevante y complementaria. Opina sobre aspectos variados del texto y explica la intención de los recursos textuales a partir de su conocimiento y experiencia.

Las habilidades-destrezas que se están en juego son:

- Relaciona los datos extraídos de gráficos, tablas y organizadores con la información central de uno o dos textos.

- Deduce el tema central y los subtemas de un texto.

- Explica por qué el autor usa determinados recursos textuales (comillas, paréntesis, ilustraciones y gráficos) en el texto.

- Opina sobre los distintos mensajes que puede extraer de los textos y de su conocimiento sobre el tema.

Como se ve los MPA actúan como un mapa mental de los profesores concretando modelos de secuencia instruccionales centrados en el aprendizaje de los estudiantes. De esta forma complementan, ordenan y secuencian el DCN vigente. En ninguna parte aparece el proceso que debe seguir el estudiante para aprender a aprender desarrollando habilidades mentales-destrezas.

Aunque los MPA secuencian los contenidos por grados no secuencian las habilidades -destrezas- necesarias para aprender los contenidos y menos aún habla 
de valores y actitudes. En los ejemplos de MPA propuestos más arriba se puede observar que lo relacionado con la inteligencia emocional se describe como opina, expresa sus gustos y preferencias, etc. Una forma muy elemental de desarrollar valores y actitudes.

Los MPA homogenizan el sistema educativo, pero la selección de algunas habilidades y contenidos concretos impulsan a los profesores a ser selectivos, ante el riesgo de ser mal evaluados tanto ellos como sus estudiantes. Al aplicar las habilidades -destrezas- a determinados contenidos de cada área, corre el riesgo de que algunos contenidos queden fuera, reduciéndose así el volumen de contenidos aprendidos por los estudiantes.

3.2.2. Forster, M. (2007) define los MPA como instrumentos de aprendizaje y control que articulan "lo que los estudiantes saben, comprenden y saben hacer" y esto permite convertirlos en indicadores y descriptores del aprendizaje.

- Al remitirnos a lo que "saben" estamos refiriéndonos a seleccionar contenidos relevantes en función de la evaluación externa.

- El hecho de "comprender" lo asociamos a capacidades-destrezas-habilidades entendidas como herramientas mentales que utiliza el estudiante para aprender. Se refieren al "para qué" de la educación en la Sociedad del Conocimiento. Este planteamiento es, a nuestro parecer, correcto, pero habría que mejorar la selección y secuenciación de las habilidades-destrezas que se han colocado en los MPA.

- El "son capaces de hacer" nos remite a los métodos y estos, aun suponiendo que se refieran a los métodos de aprendizaje, no están claros -por no decir que no aparecen- en los MPA.

La contradicción notable entre la concepción de "progreso del aprendizaje" y "resultados de aprendizaje" es que se permite hablar de procesos educativos visibles mientras se habla de productos educativos artificialmente construidos. Un diseño pedagógico es visible y puede mostrar, a medida que el tiempo pasa, un progreso en lo que un estudiante puede hacer. Una prueba estandarizada construye limitadamente una idea sobre lo que un estudiante puede hacer en un momento dado. Si bien los MPA se articulan en torno a estándares, la noción de que existe un progreso del aprendizaje es nueva en el currículum nacional formal. (Salinas, 2012, 30). Según este autor, hasta el 2012, no existe, en la literatura revisada, un programa o reporte de investigación que analice el impacto del uso de los MPA en las aulas chilenas que es el país de América Latina en el que se lleva trabajando durante varios años con los MPA.

La aproximación al problema del "progreso" en el aprendizaje se construyó con una variedad de observaciones empíricas iterativas de variados modos de desempeño, buscando describir cómo aprenden los estudiantes. No se trata de describir cómo es que los estudiantes debieran aprender y demostrar lo que han aprendido, sino que es la interpretación de los desempeños la que construye la progresión, todo en base a una articulación teórica y operacional más o menos explícita. Pero 
para que este tipo de investigaciones tengan un efecto en el aula, lo primordial es la confianza en el saber profesional docente. (p. 31) Algo que dudamos en el Perú, analizando los datos de las evaluaciones censales de los profesores de los años 2007 y 2008.

\subsection{Las competencias-capacidades-habilidades en los MPA}

Falta el desarrollo teórico que sustente el cómo se tiene que intervenir en el aula para desarrollar competencias-capacidades. Se han dado orientaciones prácticas sobre la intervención en el aula durante las sesiones de aprendizaje, que, en muchos casos, son más un impedimento que una ayuda para la creatividad de los profesores bien preparados. Se programa para cumplir un requisito administrativo y en función de él, no en función de las necesidades de los estudiantes y su progreso cognitivo. Por otra parte, cómo van a desarrollar los estudiantes las destrezas, si nadie les explica qué son tales destrezas -definición conceptual- y los procesos para desarrollarlas, ni tampoco los métodos de aprendizaje...

Suponemos que las competencias clave se refieren a las capacidades fundamentales (potencialidades que debe desarrollar-adquirir el estudiante para poder aprender contenidos) como comprensión, expresión, orientación espacio-temporal, socialización, etc.) y también a las capacidades superiores como pensamiento crítico, creativo, resolutivo y ejecutivo.

Analizando los MPA y la RA se puede observar que en ninguno de los dos aparecen los valores-actitudes. Parece que se dan por supuestos. Es una falencia que impide el enganche con lo indicado en el DCN (2008).

\section{RUTAS DE APRENDIZAJE (RA)}

Como ya hemos indicado antes el MINEDU (2013) define las RA como un conjunto de herramientas que proponen orientaciones pedagógicas y sugerencias didácticas para la enseñanza efectiva de los aprendizajes fundamentales. Las rutas se han construido a partir de los mapas de progreso que expresan los estándares de desempeño que debe lograr cada estudiante al término de cada ciclo de la educación básica regular

Tienen por finalidad orientar el trabajo de los docentes para conseguir que sus estudiantes consigan los estándares de calidad que exigen los mapas de progreso. Como las competencias son "aprendizajes complejos" las orientaciones proporcionadas por las rutas de aprendizaje posibilitan que todos los docentes de la misma área trabajen las mismas capacidades y destrezas para adquirir los aprendizajes fundamentales.

También permite recibir orientación sobre la forma de evaluar por competencias tanto en las evaluaciones inicial y de proceso, como la final. Además posibilita realizar una intervención pedagógica en el aula que permita el desarrollo de capacidades y competencias y el aprendizaje de los contenidos fundamentales. 
Hasta el primer semestre del año 2013 se han implementado rutas de aprendizaje de tres áreas: Comunicación, Matemáticas y Educación para la ciudadanía.

Leyendo tales rutas de progreso se puede observar que en muchos casos las habilidades que propone en cada ciclo -destrezas- no están claras ni secuenciadas; por otra parte son escasas y las actividades presentadas son muy elementales. De igual forma los MPA presentan como destrezas que dudo que lo sean; por ejemplo: comprender -la comprensión es una capacidad fundamental, no una destreza-, opinar, etc. Como ya hemos indicado, ya que las Rutas de aprendizaje presentan menos destrezas que las que tiene el DCN -solo aparecen las más sencillas y elementales- nos exponemos a que el profesor caiga en la tentación de trabajar estas dejando todas las demás, que también están prescritas.

No olvidar, por otra parte, que las RA no son recetas que hay que seguir de manera ciega y rígida; por el contrario, ofrecen orientaciones que cada docente podrá adecuar a su realidad, haciendo uso de sus saberes pedagógicos y de su creatividad.

El MINEDU ha creado el portal Perú Educa, que es un espacio virtual para todos los docentes, directores, estudiantes, padres de familia y otros actores educativos. Este portal presenta una serie de actividades de auto-evaluación a través de las cuales el estudiante puede comprobar sus progresos en el aprendizaje. Así mismo puede proporcionar inspiración al docente para proponer actividades en las diversas áreas de aprendizaje. Son interesantes, progresivas e interactivas. Pueden servir de modelo para que el docente realice actividades de aprendizaje en la clase, siempre que impliquen el desarrollo completo de los ejercicios y no indicar solo la respuesta. Está disponible en: www.perueduca.pe

\section{MAPAS DE PROGRESO DEL APRENDIZAJE Y PARADIGMA SOCIO- COGNITIVO-HUMANISTA}

Por las razones expuestas más arriba creemos que la propuesta de trabajar con los MPA es manifiestamente mejorable. El paradigma socio-cognitivo-humanista desarrollado y utilizado por la Universidad Marcelino Champagnat está en la misma línea que los MPA teniendo en cuenta las acotaciones que hemos hecho antes y añadiendo el marco teórico y metodológico-práctico que permite que los estudiantes desarrollen capacidades y competencias y valores y actitudes y aprendan contenidos curriculares. Por otra parte, supera las dificultades a las que se enfrentan los MPA al posibilitar que el docente seleccione las destrezas más adecuadas para su contexto y área, así como los valores-actitudes más necesarios. Ver los libros publicados en la misma Universidad (Latorre Ariño, M. y Seco del Pozo, C. J., 2010a; 2010b; 2010c; 2010d; 2010e; 2013; Latorre Ariño, M., 2013).

El paradigma cognitivo coloca al estudiante como protagonista del aprendizaje, ya que explicita cómo aprende el que aprende, qué procesos utiliza al aprender, qué capacidades-destrezas necesita para aprender y seguir aprendiendo. Es socialcontextual porque tiene en cuenta que el estudiante aprende en un escenario con- 
creto, lleno de interacciones, en el que existe un modelo de cultura, pues "lo que nos rodea nos socializa; tomar conciencia y posición sobre lo que nos rodea nos educa”. (Pérez Gómez, A. I., 2007)

El desarrollo integral y armónico de la persona consiste en integrar y desarrollar en la misma los elementos básicos de la cultura global y local-institucional en el marco de la cultura escolar. La educación integral se logra desarrollando armónicamente capacidades-destrezas, valores-actitudes, conocimientos y procedimientos (métodos, estrategias y técnicas de aprendizaje). De esta manera entramos en un modelo de aprendizaje constructivo, significativo y funcional.

La educación integral exige un modelo de planificación que facilite la integración de capacidades-valores -como fines que se quieren conseguir-, utilizando como medios los contenidos curriculares y los métodos. El Modelo T -que es un marco conceptual- permite integrar estos elementos de manera orgánica y se convierte en puerta de entrada en la sociedad del conocimiento (Román, M. y Díez Pérez, E. 2008, 72-73).

Creemos que es indispensable que docentes y estudiantes, definan conjuntamente los conceptos (capacidades-destrezas, competencias, valores-actitudes) que se utilizan en el trabajo de aula y conozcan los procesos mentales que se deben utilizar para conseguir capacidades-competencias y aprender contenidos. Si no es así, tanto los docentes como los estudiantes, realizarán actividades y tareas pero sin comprender su sentido y utilidad. De nuevo estamos en el problema de "poseer abundancia de medios tecnológicos -bienvenidos sean en las aulas- y confusión en los fines" (Rojas, E.).

Para conseguir todo lo indicado hay que elaborar las programaciones anuales o bianuales (ciclo) y Unidades de Aprendizaje con las actividades de la forma siguiente:

- Se realiza el panel de Capacidades-destrezas del área correspondiente.

- Se identifican y expresan las competencias relacionadas con las capacidades elegidas ("el núcleo de una competencia es una capacidad").

- Se disgregan las destrezas por niveles de aprendizaje.

- Se definen todos conceptos anteriores y se definen los procesos mentales de cada destreza.

- Se incorpora el panel de valores y actitudes propios de la I. E. (Aquí se pueden incluir los temas o contenidos transversales también).

- Se identifican los contenidos del grado y área ordenándolos de forma arquitectónica (por bloques y temas).

- Se identifican los métodos de aprendizaje, relacionados con las destrezas elegidas en el panel de capacidades-destrezas del grado-área. 
A partir de aquí surgirán las Unidades de Aprendizaje con las actividades que van a realizarse en el aula, para aprender los contenidos propios de la misma y desarrollando las capacidades-destrezas y los valores-actitudes.

Las actividades son estrategias de aprendizaje que diseña el docente (mediador), expresadas en forma de resolución de situaciones en contextos lo más próximo a la realidad del estudiante, para que estos desarrollen capacidades-destrezas, valoresactitudes y aprendan los contenidos propios del área. En consecuencia para que adquieran competencias competentes. (Román y Díez, 2008, 84)

Cualquier modelo de actividad similar a las que se han trabajado en el aula puede convertirse en una prueba de evaluación de proceso o final, incluyendo las matrices de evaluación correspondientes.

El modelo de los cuatro pasos pedagógicos (Latorre, M. y Seco, C. J. 2013, 33) desarrollado en nuestra universidad garantiza la actividad del estudiante en el proceso de aprendizaje y que este sea significativo y funcional.

Este proceso garantiza el desarrollo de capacidades-destrezas y valores-actitudes y el progresivo aprendizaje de los contenidos utilizando los métodos adecuados. Al mismo tiempo desarrolla la inteligencia del estudiante, considerada como un conjunto de procesos cognitivos (capacidades, destrezas y habilidades), conjunto de procesos afectivos (valores, actitudes y micro-actitudes) y un conjunto de esquemas mentales (arquitectura del conocimiento, en forma de marcos, redes y mapas conceptuales, mentales, esquemas gráficos de distinto tipo, etc.) (Díez López, E. 2006, 184).

En realidad los MPA y el Modelo T actúan como mapas mentales del profesor para impulsar el aprendizaje de los estudiantes.

\section{6. ¿CUÁl ES EL ROL DEL DOCENTE EN EL PROCESO DE APRENDIZAJE POR CAPACIDADES Y COMPETENCIAS?}

El desarrollo de las capacidades y el logro de las competencias, requiere un docente que intente "lograr que sus estudiantes aprendan de manera significativa y funcional desarrollando habilidades mentales y actitudes emocionales".

Solo se produce aprendizaje cuando el estudiante aprehende y estructura la información recibida incorporándola significativamente a sus contenidos previos; para ellos debe plantearse conflictos cognitivos adecuados y resolverlos. La actividad mental -neuronal- es indispensable y la interacción entre iguales facilita el aprendizaje.

Para conseguir lo dicho la actividad del docente deja de ser central y queda sustituida por la centralidad del estudiante y su aprendizaje. Esto lo logra en la medida en que el docente:

- posibilita el protagonismo del estudiante para que este pueda jugar un papel activo en su propio proceso de aprendizaje. 
- el docente asume el papel de mediador o facilitador de la construcción sociocognitiva del conocimiento por parte del estudiante, ofreciendo oportunidades de aprendizaje, espacios para la discusión y la reflexión, materiales educativos, retroalimentación, funcionalidad de los aprendizajes, etc.

- el docente diseña creativamente actividades que desarrollan capacidades y valores en los estudiantes.

- el docente se preocupa porque todos sus estudiantes logren aprendizajes significativos.

Todo lo indicado anteriormente significa que el docente realiza una labor centrada en el aprendizaje del estudiante. Estos profesores consiguen:

- Involucrar a un número mayor de sus estudiantes en un proceso de aprendizaje efectivo.

- Lograr que sus estudiantes aprendan mejor.

- Brindar una formación integral con calidad y calidez humana.

- Desarrollar habilidades de reflexión y crítica de su propia práctica docente.

- Desarrollar sesiones de aprendizaje, utilizando las metodologías activas y didácticas centradas en el aprendizaje del estudiante.

En conclusión, aunque el estudiante es el protagonista de su propio aprendizaje, el profesor es el "catalizador-mediador" que facilita que la reacción -el proceso de aprendizaje- se desencadene y avance con rapidez, pues estamos convencidos de que "la calidad de un sistema educativo tiene como techo la calidad de sus docentes". (Barber, M. y Mourshed, M. 2008, 15)

\section{REFERENCIAS BIBLIOGRÁFICAS}

Barber, M. y Mourshed, M. (2008). Cómo hicieron los sistemas educativos con mejor desempeño del mundo para alcanzar sus objetivos. Informe McKinsey (2007). Santiago de Chile, Chile: PREAL, N. ${ }^{\circ}$ 41, 2008.

Boutang, Y. M., Corsani, A. \& Lazzarato, M. (2004). Capitalismo cognitivo: propiedad intelectual y creación colectiva. Madrid, España: Traficante de Sueños.

Bransford, J. D., Brown, A. L. \& Cocking, R. R. (2000). How people learn: brain, mind, experience, and school (Expanded ed.). Washington, DC: National Academy Press.

Cassasus, J. (2010). Las reformas basadas en estándares: un camino equivocado. En J. P. Valenzuela, C. Bellei, D. Contreras (Eds.), Ecos de la Revolución Pingüina: Avances, Debates y Silencios en la Reforma Educacional (pp. 85-110). Santiago, Chile: Universidad de Chile.

Díez López, E. (2006). La inteligencia escolar. Aplicaciones al aula. Una nueva teoría para una nueva sociedad. Santiago de Chile, Chile: Arrayán Editores. 
Docencia. (2009). Estandarización educativa en Chile: Un peligroso hábito. Docencia, $38,4-17$.

Forster, M. (2007). Argumentos en favor de los mapas de progreso en Chile. IX Conferencia Internacional UKFIET sobre Educación y Desarrollo en Septiembre del 2007.

http://es.scribd.com/doc/84773499/Orientacion-Uso-Mapas-de-Progreso

http://ipeba.gob.pe/images/stories/CHILE_argumentos_favor_mapas_progreso.pdf

Latorre Ariño, M. y Seco del Pozo, C. (2010a). Diseño curricular nuevo para una nueva sociedad. Teoría: qué cambiar y cómo cambiar. Lima, Perú: Universidad Marcelino Champagnat.

Latorre Ariño, M. y Seco del Pozo, C. (2010b). Diseño curricular nuevo para una nueva sociedad. Programación y evaluación de Educación Inicial. Lima, Perú: Universidad Marcelino Champagnat.

Latorre Ariño, M. y Seco del Pozo, C. (2010c). Diseño curricular nuevo para una nueva sociedad. Programación y evaluación de Educación Primaria. Lima, Perú: Universidad Marcelino Champagnat.

Latorre Ariño, M. y Seco del Pozo, C. (2010d). Diseño curricular nuevo para una nueva sociedad. Programación y evaluación de Educación Secundaria. Lima, Perú: Universidad Marcelino Champagnat.

Latorre Ariño, M. y Seco del Pozo, C. (2010e). Paradigma Socio-cognitivo-humanista. Desarrollo y evaluación de Capacidades y valores en la Sociedad del Conocimiento para "aprender a aprender". Lima, Perú: Universidad Marcelino Champagnat.

Latorre Ariño, M. y Seco del Pozo, C. (2013). Metodología. Estrategias y técnicas metodológicas. Lima, Perú: Universidad Marcelino Champagnat.

Latorre Ariño, M. (2013). Diseño curricular por capacidades y competencias en Educación Superior. Lima, Perú: Universidad Marcelino Champagnat.

MINEDU (2013). Ministerio de Educación. Directiva "Normas y Orientaciones para el Desarrollo del Año Escolar 2013".

Mineduc. (n.d.). Mapas de Progreso del Aprendizaje: Sector Ciencias Naturales, Mapa de Progreso de Materia y sus Transformaciones. Santiago, Chile.

Ministerio de Educación de Chile (2008). Orientaciones para el uso de los Mapas de Progreso del Aprendizaje. Un material de apoyo para el trabajo docente. Gobierno de Chile. Santiago: Editorial Valente Ltda.

Pérez Gómez, A. I. (2007). La naturaleza de las competencias básicas y sus aplicaciones pedagógicas. Cuadernos de Educación de Cantabria. Cantabria, España: Consejería de Educación.

Román Pérez, M. y Díez López, E. (2008). Diseño curricular de aula: Modelo T, Puerta de entrada en la Sociedad del Conocimiento. Santiago de Chile, Chile: Editorial Conocimiento.

Salinas, I. (2012). Mapas de progreso ¿del aprendizaje? En Revista Docencia, N. ${ }^{\circ} 47$, agosto 2012 . 